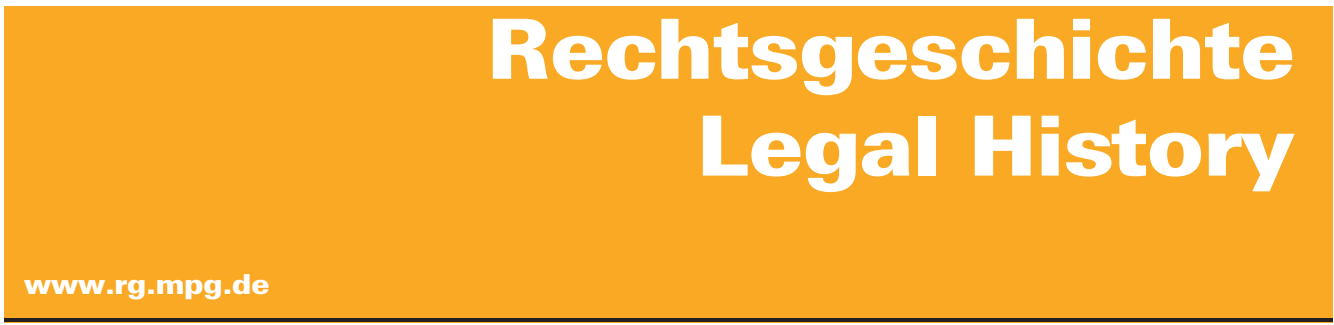

http://www.rg-rechtsgeschichte.de/rg23

$\operatorname{Rg} 232015 \quad 174-184$

Zitiervorschlag: Rechtsgeschichte - Legal History Rg 23 (2015)

http://dx.doi.org/10.12946/rg23/174-184

\title{
Wilfried Hartmann
}

Synoden schaffen Räume: Metropolen, Diözesen und Pfarreien in den Synodalkanones des 9. Jahrhunderts 


\section{Abstract}

This study on »space in canon law« investigates the concepts of provincial, diocesis and parrocchia in the synodal decisions and the capitularies of bishops in the ninth century. The space of provincia was not precisely determined at the end of this age. Perhaps not even the provincial council is differentiated from a diocesan synod. The space of the diocese in the ninth century is best known by the trip of visitation that the bishops had undertaken since late Antiquity. In his capitularies, the bishop speaks to all members of his diocese, clergy and laity, and he provides them precepts and prohibitions. The new significance of the diocese is also made clear by the prohibition of transmigration and transfer of bishops from one diocese to another, which gained new importance during the ninth century. The subdivision of a diocese into several districts of deans can already be found in the middle of this age. The smallest format of space in the canon law was the parrocchia, the parish. We must be careful in using this term, because it often also means bishopric or diocese. The parish was defined as the space where only one priest has the right to hold his office. Since the ninth century, parish and diocese are very precisely defined as particular spaces. Bishop Theodulf of Orléans (798-818) and archbishop Hincmar of Reims (845-882) made numerous remarks in their capitularies about the formation of diocese and parish. 
Wilfried Hartmann

\section{Synoden schaffen Räume: Metropolen, Diözesen und Pfarreien in den Synodalkanones des 9. Jahrhunderts}

Wenn man etwas zum Thema »Raum im Kirchenrecht" sagen will, dann muss man zuerst fragen, nach welchen Begriffen man suchen möchte, um Material zu den Raumvorstellungen auf frühmittelalterlichen Konzilien zu sammeln. Verena $\operatorname{Epp}^{\mathbf{1}}$ hat vor einigen Jahren einige Begriffe zusammengestellt, mit denen im Frühmittelalter Raum erfasst wurde, nämlich: provincia - patria civitas - terra-diocesis - parrochia.

In unseren kirchenrechtlichen Quellen bleiben davon vor allem die drei Begriffe provincia, diocesis und parrochia, mit denen die drei Ebenen der kirchlichen Raumgliederung, nämlich Kirchenprovinzen, Diözesen und Pfarreien bezeichnet worden sein könnten.

Der Begriff provincia ist in den kirchenrechtlichen Quellen nicht sehr häufig, was vielleicht etwas erstaunen mag; als Bezeichnung für »Kirchenprovinz« kommt er anscheinend äußerst selten vor. Anders ist es mit den beiden anderen Begriffen; vor allem das Wort parrochia findet sich recht häufig; es bedeutet allerdings nicht nur »Pfarrei«, sondern vielfach auch »Bistum«, »Diözese«. Dazu wird später noch einiges zu sagen sein.

Wenn im Folgenden von Synodalkanones die Rede ist, dann soll dabei die Entstehung dieser Normen keine Rolle spielen; es interessiert nicht, ob sie auf Diözesansynoden, auf Provinzialkonzilien, auf Konzilien mit Teilnehmern aus mehreren Kirchenprovinzen oder auf Landes- oder Reichskonzilien erlassen wurden. Es soll nur darauf geachtet werden, ob in den Kanones Raumbegriffe vorkommen und was über diese Räume gesagt wird.

Synodalkanones gibt es in karolingischer Zeit eigentlich erst seit 813 . Unter Pippin und auch im größten Teil der Regierungszeit Karls des Großen wurden die Beschlüsse der Synoden als Herrscher- kapitularien verkündet, d. h. sie waren keine Normen der Kirche, sondern solche der Herrscher. Diese als Kapitel in königlichen Erlassen bekanntgemachten und verbreiteten Anweisungen hatten ohne Zweifel eine reichsweite Geltung.

Die karolingischen Synodalkanones entstanden als Nachahmung der Konzilsbeschlüsse der alten Konzilien, die den Klerikern des beginnenden 9. Jahrhunderts aus den Sammlungen historischer Ordnung, besonders aus der Collectio DionysioHadriana, bekannt waren. In solchen Sammlungen, z. B. in der Collectio Hispana, konnten sie auch die Kanones der westgotischen Synoden und der Synoden des merowingischen Frankenreichs kennen lernen. Solche Synodalbeschlüsse wurden bereits am Beginn des 9. Jahrhunderts auch in systematisch geordneten Sammlungen wie etwa der Collectio Dacheriana verarbeitet. Dabei wurde kein Unterschied gemacht, welche Art von Konzilien, reichsweite oder nur partikulare, diese Normen erlassen hatte.

\section{Kirchenprovinz (provincia)}

Synodalkanones über Metropoliten und Provinzialkonzilien sind in der karolingischen Zeit sehr selten. In der Zeit Karls des Großen hatten nur einige Kapitularien die Forderung erhoben, dass zweimal jährlich eine Provinzialsynode zusammentreten sollte, so etwa in der Admonitio generalis (789) c. $13 .^{2}$ Was die Synoden der Zeit Karls angeht, so wird die Abhaltung von Provinzialsynoden einzig in der bairischen Provinzialsynode von Reisbach / Freising / Salzburg aus dem Jahr 799/800 verlangt: Zweimal im Jahr sollen solche Versammlungen zusammenkommen. ${ }^{3}$ Zwei Konzilien der Zeit Ludwigs des Frommen, ein nicht genau zu

1 Epp (1998).

2 MGH Fontes iuris Germanici antiqui

16, Mordek u. a. (2012) S. 190. Vgl.

PANGerl (2011) $170 \mathrm{f}$

3 Ebd. 171. 
datierendes aus der Zeit zwischen 818/19 und 829 (c. 1) und Paris 829 (c. 26), forderten das Zusammentreten von Provinzialsynoden wenigstens einmal im Jahr. ${ }^{4}$

Nach dem Tode Ludwigs des Frommen wurde die Abhaltung von solchen Synoden nur im Westfrankenreich gefordert: Das Konzil von MeauxParis $(845 / 46)$ befasst sich in cc. 32 und 33 mit diesem Thema:

Kapitel 32 erbittet vom König, den Metropoliten und ihren Suffraganbischöfen zu gestatten, dass sie ein- oder zweimal im Jahr zu Synoden zusammenkommen dürfen (sinodice conveniri). Die Synode verweist auf die decreta canonum, die Bestimmungen der alten Kirche (u.a. Nicaea, Chalkedon, Antiochia); etwas Neues ist damit also ganz und gar nicht eingeführt. In c. 33 werden die Bischöfe verpflichtet, an Provinzialsynoden teilzunehmen: Wer nicht durch eine offensichtliche Unmöglichkeit (evidens impossibilitas) am Erscheinen gehindert ist, muss präsent sein bei Gefahr des Amtsverlusts.

Im weiteren Verlauf des 9. Jahrhunderts kümmerte sich vor allem Erzbischof Hinkmar von Reims um die regelmäßige Veranstaltung von Provinzialsynoden. Er bittet im Synodalschreiben vom November 858 Ludwig den Deutschen darum, Konzilien zu erlauben:

Ut temporibus a sacris regulis constitutis comprovinciales synodos cum episcopis et speciales cum presbyteris habere quiete possint, annuite. ${ }^{5}$ Hier sind also sowohl Provinzialsynoden als auch Diözesansynoden gemeint. Auch die Synode von Langres 859 (c. 7) ${ }^{\mathbf{6}}$ verlangte, dass mindestens einmal pro Jahr eine Provinzialsynode abgehalten werden müsse (per singulas provincias semel per annos singulos); alle zwei Jahre (semel intra biennium) ein generalis episcoporum conventus, also eine Reichssynode. Aus dem Ostfrankenreich oder aus Italien sind solche
Forderungen aus dem 9. Jahrhundert nicht bekannt.

In seinem kürzlich erschienenen Buch über die Metropolitanverfassung des karolingischen Frankenreichs hat Carlo Daniel Pangerl sehr ausführlich die Provinzialsynoden behandelt. ${ }^{7}$ Er konnte 31 solche Synoden nachweisen von insgesamt über 150 Synoden, die zwischen dem ausgehenden 8. und dem Ende des 9. Jahrhunderts im Frankenreich nördlich der Alpen stattgefunden haben. Mindestens sechs weitere Synoden waren Synoden der Suffragane unter der Leitung ihres Metropoliten, an denen eine kleine Anzahl von weiteren Bischöfen aus einer oder zwei Nachbarprovinzen teilnahmen: Diese sog. »erweiterten« Provinzialsynoden möchte Pangerl sehr deutlich von den »echten « Provinzialsynoden trennen. ${ }^{\mathbf{8}}$

Nur von drei dieser 31 Provinzialsynoden haben sich auch Kanones erhalten; neben der bayerischen »Tripelsynode " (Reisbach / Freising/Salzburg) von $799 / 800$ handelt es sich um die Synoden von Köln 887 und von Metz 893. ${ }^{9}$ Die Beschlüsse dieser beiden letzten Synoden sind äußerst schwach überliefert; sie sind nämlich nur aus jeweils einem Druck des 16. Jahrhunderts bekannt. ${ }^{10}$ Das zeigt, dass die Reichweite dieser Beschlüsse sehr gering gewesen ist. Anders die bairische Tripelsynode: Ihre Beschlüsse wurden wohl in allen Bistümern und Klöstern der bairischen Kirchenprovinz verteilt, wie die beiden noch erhaltenen Handschriften bezeugen: Clm 28135 aus der Dombibliothek Freising, die kurz nach 800 entstanden ist, und Clm 6333 aus Kloster Benediktbeuern, ebenfalls um 800 geschrieben. Das heißt doch: Unter Erzbischof Arn von Salzburg wurde die Kirchenprovinz als Raum wahrgenommen und realisiert!

Aus der Zeit Karls des Großen kennen wir allerdings nur Provinzialkonzilien aus der Kirchenprovinz Salzburg; erst nach dem Tod Karls gab es

4 Ebd. 173.

5 MGH Concilia 3 (Hartmann 1984)

S. 414, 7-9.

6 Ebd. S. 477

7 Pangerl (2011) 160-260. Dieses

Kapitel umfasst fast ein Drittel des Buches.

8 Vgl. ebd. $162 \mathrm{f}$.

9 Ebd. 241.

10 Vgl. MGH Concilia 5 (HartmanN u. a. 2012) S. 212 und 308 und PANGerl (2011) 241. 
dann - allerdings sehr wenige - Provinzialsynoden auch in anderen Kirchenprovinzen: bis 840, also bis zum Tod Ludwigs des Frommen, lediglich zwei (814 und 822 in den Provinzen Reims und Sens). Erst nach 840 nimmt die Anzahl dieser Synoden stark zu, bleibt aber immer noch recht überschaubar.

Obwohl im karolingischen Frankenreich immer wieder die jährliche oder wenigstens zweijährige Abhaltung von Provinzialkonzilien gefordert wurde, ${ }^{11}$ haben also solche Synoden nur recht selten stattgefunden; für ungefähr die Hälfte der Kirchenprovinzen des Frankenreichs sind überhaupt keine derartigen Synoden bezeugt. Allein drei Metropoliten haben fast die Hälfte der bekannten Synoden dieses Typs zusammengerufen: Arn von Salzburg (785-821) und Wenilo von Sens (837/38-865) je 5, Arnustus von Narbonne (um 896-912/13) 4 und zusätzlich noch 2 erweiterte Provinzialsynoden. Interessanterweise verteilen sich die Sedenzzeiten dieser drei Erzbischöfe über die gesamte Karolingerzeit, d.h. die Begeisterung für die Einberufung von Synoden der Suffragane eines Metropoliten war nicht an bestimmte Zeiten gebunden, sondern hing weitgehend von der Person des Metropoliten ab.

Nach Pangerl hatten sich nur eine Provinzialsynode als synodus provincialis und zwei weitere als synodus comprovincialis bezeichnet. ${ }^{\mathbf{1 2}}$ Nicht gesehen hat Pangerl, dass auch die Synode von Metz 893 sich in c. 1 synodus comprovincialis nennt. ${ }^{13}$ Pangerl untersuchte nicht, ob auch solche Konzilien, die nach seinen strengen Kriterien eigentlich keine Provinzialsynoden sind, in den zeitgenössischen Quellen synodus (com-)provincialis genannt werden. Dies ist aber der Fall: Erzbischof Hinkmar von Reims benutzte den Begriff synodus comprovincialis, um eine Synode zu bezeichnen, auf der mehrere Metropoliten und Bischöfe aus mehreren Kirchenprovinzen zusammenkamen. ${ }^{\mathbf{1 4}}$ Der Terminus für Provinzialsynode ist bei Hinkmar: synodus provincialis. ${ }^{\mathbf{1 5}}$

Kann man aus dem Inhalt der Kanones von Provinzialsynoden etwas über die Besonderheit dieser Synoden sagen? Wenn man die 46 Kanones der Tripelsynode Arns von 799/800 betrachtet, dann erkennt man, dass eine größere Anzahl von diesen aus der Admonitio generalis 789 und aus der Synode von Frankfurt 794 übernommen sind, also aus Reichssynoden bzw. Kapitularien des Frankenkönigs. ${ }^{16}$ Daneben aber gibt es auch Bestimmungen mit regionalem Bezug oder solche, die an Diözesansynoden erinnern. ${ }^{\mathbf{1 7}}$ Jedenfalls erscheint das Provinzialkonzil hier als Schaltstelle zwischen der Zentrale und der Peripherie. Dagegen sind die Kanones von Köln 887 und von Metz 893 ganz normale Reformkanones. ${ }^{18}$ Hier kann man fragen: Warum werden auf einer Provinzialsynode Kanones erlassen, die eigentlich auf eine Reichssynode gehören?

Für Köln 887 ist das vielleicht zu erklären: In der Präfatio heißt es nämlich, die Synode sei cum consensu gloriosissimi imperatoris Caroli (= Karl III.) et Mogontini sedis archiepiscopi Liudberti zusammengetreten. ${ }^{19}$ Das kann man doch so deuten, dass Erzbischof Willibert von Köln nicht souverän über die Einberufung einer Provinzialsynode entscheiden konnte und vor allem, dass die auf der Synode erlassenen Kanones nur gültig waren durch die Zustimmung des Kaisers und des Mainzer Erzbischofs. $^{20}$

Kapitel 1 von Metz 893 erklärt: ... tanto tempore transacto comprovincialem synodum non habuimus ${ }^{\mathbf{2 1}}$ - und tatsächlich: Aus der Kirchenprovinz Trier ist aus dem gesamten 9. Jahrhundert keine andere Provinzialsynode bekannt. ${ }^{22}$ Und diese Synode
11 Vor allem in Meaux-Paris 845/46 c. 32, MGH Concilia 3 (HaRtmann 1984) S. 100, und in Langres 859 c. 7 (ebd. S. 477), vgl. Pangerl (2011) 251 mit Anm. 316.

12 Pangerl (2011) 246 f. mit Anm. 298.

13 MGH Concilia 5 (Hartmann u.a. 2012) S. 309, 21.

14 Vgl. Register zu MGH Concilia 4 (HartManN 1998), bes. S. 57, 16; S. 436, 5 f.; S. 452, 28; 497,7; S. 503, 34 und S. 504, 12.20.14.

15 Ebd. S. 91, 21; S. 395, 4.20; S. 444, 9.24; S. 544, 39 f. und S. 555, 24.
16 Vgl. MGH Concilia 2,1

(Werminghoff 1906) S. 208-213.

17 Vgl. ebd. c. 20-23 (S. 210).

18 Vgl. MGH Concilia 5 (HartmanN

u. a. 2012) S. 213-215 und

S. 309-313.

19 Ebd. S. 212, 32 f.

20 Pangerl (2011) hat all dies überhaupt nicht thematisiert.

21 MGH Concilia 5 (Hartmann u. a. 2012) S. 309, $20 \mathrm{f}$.

22 Pangerl (2011) 220. 
greift in mehreren Kapiteln (c. 3, 5, 6, 8 und 12) auf die Admonitio synodalis zurück, also einen Text, der sonst nicht auf Konzilien als Autorität herangezogen wird, sondern eher in Bischofskapitularien oder auf Diözesansynoden. Denn diese Admonitio ist eine Ansprache eines Bischofs zu seinen Priestern. Dies kann als Zeugnis für ein Vermischen der Formen dienen, die im 9. Jh. keineswegs schon so gefestigt sind, dass wir ganz klar sagen können: Das ist eine Provinzialsynode, das eine Diözesansynode, das eine Reichssynode.

Man wird wohl aus dieser Unsicherheit auch schließen dürfen, dass der Raum provincia - Kirchenprovinz auch am Ende des 9. Jahrhunderts noch keineswegs eindeutig bestimmt und ihre Grenze erkennbar und fest war!

$$
* * *
$$

\section{Diözesen}

Auch die Diözese als Raum wird zuerst durch die Diözesansynoden ${ }^{23}$ beschrieben, auf denen der Bischof mit seinen Priestern zusammenkommt. Als älteste sicher bezeugte Diözesansynode gilt die Synode von Auxerre im Jahr 585, deren Akten nach der Subskription des Bischofs Unacharius von Auxerre die Unterschriften von 7 Äbten, 34 Priestern und 3 Diakonen tragen. ${ }^{24}$ Kapitel 7 dieser Synode schreibt vor, dass künftig jedes Jahr Mitte Mai alle Priester und am 1. November alle Äbte sich zu einer Synode in der Bischofsstadt versammeln sollen. ${ }^{25}$ Auch die westgotische Kirche kennt die Pflicht Diözesansynoden zu veranstalten. ${ }^{\mathbf{2 6}}$

Unter dem Einfluss des Bonifatius ist dann im sog. Concilium Germanicum (742) und im Konzil von 747 die Abhaltung von Diözesansynoden angeordnet worden, und auch König Pippin ließ in der Synode von Ver (755) formulieren: Et omnes presbyteri ad concilium episcopi sui conveniant (Ver, c. 8). ${ }^{27}$ Danach gibt es erst wieder im Konzil von Arles (813) c. 4 einen schwachen Hinweis auf die
Diözesansynode, wenn - innerhalb eines Zitats aus Toledo IV (633) c. 26 - davon die Rede ist, dass Priester zur Synode kommen (ad concilium venerint), um ihrem Bischof Rechenschaft zu geben (rationem episcopo suo reddant). ${ }^{\mathbf{2 8}}$

Ansonsten findet sich Begriff und Sache der Diözesansynode vor allem in einigen Bischofskapitularien, so bei Theodulf von Orléans, c. 4: Quando more solito ad synodum convenitis ... ${ }^{29}$ und in c. 28 : Cum vero, domino opitulante, ad synodum in unum convenerimus, ... ${ }^{30}$ Etwas später heißt es dann bei Herard von Tours (858) c. 91: Ut bis in anno concilia celebrentur ... ${ }^{31}$ Im 1. Bischofskapitular Hinkmars von Reims lautet die Bezeichnung für die Diözesansynode: Conventus presbyterorum. ${ }^{32}$ Dieser Begriff kommt aber in den anderen vier Bischofskapitularien Hinkmars nicht vor.

Belege für Diözesansynoden finden sich auch in den Freisinger Traditionen, wo solche Versammlungen als synodus publicus bezeichnet werden. ${ }^{33}$ Sie tagten sowohl im Frühjahr als auch im Herbst.

In c. 9 der Synode von Toulouse (844) wird davon gesprochen, dass nicht öfter als zweimal pro Jahr Diözesansynoden stattfinden sollen. ${ }^{\mathbf{3 4}}$ Damit soll vor allem die Bedrückung der Landpriester durch den Bischof und seine Amtsträger (ministri) verhindert werden.

In dem bereits zitierten Abschnitt aus Kapitel 7 von Quierzy 858 ist auch davon die Rede, dass es synodi speciales geben soll, in denen die Bischöfe cum presbyteris zusammentreten. ${ }^{35}$ Damit sind offensichtlich die Diözesansynoden gemeint, die periodisch abgehalten werden sollen. ${ }^{36}$

Weiterhin wurde der Raum der Diözese durch die Visitationsreisen für den Bischof und seine Umgebung erfahrbar. Die bischöfliche Visitation, die in Abständen stattfindende Reise des Bischofs durch seine Diözese, war kirchenrechtlich eine alte Forderung. Im Westen wurde sie zuerst in Kapitel 8 des Konzils von Tarragona (516) verlangt: Jedes Jahr sollen die Bischöfe die Kirchen ihres Sprengels besuchen, um ihren baulichen Zustand zu kontrol-
23 Vgl. dazu Hinschius (1883) $582 \mathrm{ff}$.

24 MGH Concilia 1 (MAASSEN 1893)

S. 184.

25 Ebd. S. 180, 9 f.

26 Hinschius (1883) 584 Anm. 3.

27 MGH Capitularia 1 (Boretius 1883) S. 35, 2. Vgl. Barion (1931) $36 \mathrm{f}$.

28 MGH Concilia 2,1 (Werminghoff 1906) S. $250,36-$ S. $251,3$.
29 MGH Capitula episcoporum 1 (BROMMER 1984) S. 106, 9.

30 Ebd. S. 125, 10.

31 MGH Capitula episcoporum 2 Pokorny / Stratmann 1995) S. 147, 8.

32 Ebd. S. 34, 2.

33 Vgl. Hauck (1935) 245 Anm. 3.

34 MGH Concilia 3 (Hartmann 1984) S. 23.
35 Ebd. S. 414, 8.

36 Tatsächlich abgehaltene Diözesansynoden hat BARION (1931) $37 \mathrm{f}$. mit Anm. 60 zusammengestellt. 
lieren. ${ }^{37}$ Das 7 . Konzil von Toledo (646) c. 4 regelte die Kosten der Visitation. ${ }^{38}$ In der karolingischen Zeit befasste sich vor allem das Konzil von Toulouse (844) mit der Visitationsreise des Bischofs. ${ }^{39}$ Das war kaum zufällig in einer Region, die in der Nähe von Spanien lag! Wohl davon beeinflusst gehen auch die Kapitel 28 und 29 von Meaux-Paris (845/46) auf die Visitationspflicht der Bischöfe ein. Dabei beklagt c. 29, dass die pflichtgemäße Visitation der Diözesen von manchen Bischöfen raro aut nunquam durchgeführt werde. ${ }^{40}$

Seit der Mitte des 9. Jahrhunderts gab es dann noch eine weitere Verpflichtung des Diözesanbischofs, die er durch einen Besuch sämtlicher Pfarreien seiner Diözese erfüllen musste, nämlich die Abhaltung des Sendgerichts, die gerichtliche Untersuchung von Verfehlungen der Kleriker und der Laien in den einzelnen Dörfern. Für die Praxis des Sendgerichts von besonderem Interesse ist ein Kanon, dessen Entstehungszeit bisher nicht geklärt ist und in dem es heißt:

Wenn der Bischof seine Diözese bereist, soll der Archidiakon oder der Archipresbyter ein oder zwei Tage zuvor in die Pfarrgemeinden (per parrochias) gehen, die dieser besuchen will, das Volk zusammenrufen, ihm die Ankunft seines Hirten ankündigen, kraft der Autorität der heiligen Kanones auf jede Weise vorschreiben, dass alle an seinem Sendgericht am dafür bestimmten Tag teilnehmen sollen, und mit Nachdruck bekannt geben, dass jeder, der ohne schwerwiegenden Grund fehlt, ohne Zweifel aus der christlichen Gemeinschaft ausgestoßen werden muss. Dann soll er die Priester bei sich versammeln, die an jenem Ort im Dienst des Bischofs stehen, und sich um die Korrektur kleinerer und leichterer Vergehen bemühen, damit der Bischof bei seiner Ankunft auf keinen Fall durch allzu geringfügige Geschäfte ermüdet wird oder genötigt ist, sich dort länger aufzuhalten, als es die Kosten zulassen. ${ }^{\mathbf{4 1}}$

Zeit und Ort der Entstehung dieses Kanons sind unklar; aber es gibt einige Kanones, die von der Überwachung der Gläubigen und von der Überwachung der Büßer in Städten und Dörfern sprechen und die klar einer bestimmten Synode zuzuordnen sind, wie etwa die Kapitel 6 und 7 von Pavia 850. ${ }^{42}$ Dabei wurde auch das Problem wahrgenommen, dass es zu Schwierigkeiten kommen könnte, wenn Büßer in mehreren Diözesen begütert sind. ${ }^{43}$

Einige Jahrzehnte später stellte die Synode von Ravenna 898 fest:

Die Bischöfe der einzelnen Städte haben in ihrer Diözese die unbeschränkte Macht, Unzucht und Verbrechen aufzuspüren, zu verfolgen und zu bestrafen, gemäß den alten Kanones, ohne dass sie dabei von jemand gehindert werden dürfen. ${ }^{44}$

Dies sind Normen aus Italien, aber auch für Lotharingien sind die bischöflichen Sendgerichte spätestens aus der Zeit um 900 belegt, wie vor allem das Sendhandbuch des Regino von Prüm zeigt. Und für das Ostfrankenreich kann das Zeugnis des Kanons 23 von Mainz 888 angeführt werden, in dem es heißt: Itaque decernimus, ut iuxta divine legis sanctionem et secundum ipsius sedem civitatis, in qua ratio ventilatur, unaquaeque gens et cuncta adiacens parochia subiaceat, ita ut auctoritate episcopi aut iudicii examine aut sacramenti protestatione se expurget ${ }^{45}$ (es geht um Ehebrecher). Während Stefan Weinfurter angenommen hat, die Überwachung der Gläubigen wegen ehelicher Verfehlungen habe erst im 12. Jahrhundert einge-
37 Vives (1963) $36 \mathrm{f}$.

38 Diez/Rodriguez (1992) $351 \mathrm{f}$

39 MGH Concilia 3 (Hartmann 1984)

S. 18-23. Ebd. S. 21 Anm. 17 ist Li-

teratur zu diesem Thema zusam-

mengestellt.

40 Ebd. S. 99, $22 \mathrm{f}$

41 MGH Concilia 5 (Hartmann u.a. 2012) S. 590, 1-10.

42 MGH Concilia 3 (Hartmann 1984) S. $222 \mathrm{f}$.

43 Vgl. Pavia 850, c. 11 (ebd. S. 225).
44 MGH Concilia 5 (Hartmann u.a. 2012) S. 439, 25-27. 45 Ebd. S. 262, 20-23. 
setzt, ${ }^{\mathbf{4 6}}$ wurde durch das Sendgericht, das seit der Zeit um 900 bezeugt ist, bereits eine Kontrolle der Lebensführung der Gläubigen praktiziert. ${ }^{47}$

Weitere wichtige Quellen für die Konstitution der Diözesen waren die Bischofskapitularien, die seit ca. 800 im ganzen Frankenreich entstanden sind. ${ }^{48}$ Es waren zuerst einige enge Mitarbeiter Karls des Großen, die Normen für die Priester ihrer Diözese erlassen haben. Am Beginn steht Bischof Ghaerbald von Lüttich, der zwischen 802 und 809 zwei solche Texte ergehen ließ (Ghaerbald II und III). ${ }^{49}$ In Ghaerbald II heißt es mehrfach infra nostra parrochia (im Sinne von: in unserer Diözese) (in cc. 5, 12, 13 und 14), ${ }^{50}$ in c. 7 von Ghaerbald III ist von parrochia nostra die Rede und in c. 18 von de aliena parrochia. ${ }^{51}$ Ghaerbald II und III ergänzen sich gut: Während sich Ghaerbald II vor allem an die Laien wendet, gibt Ghaerbald III Regeln für die Kleriker.

Noch vor 813 entstand das später sehr weit verbreitete Bischofskapitular Theodulfs von Orléans, in dem es einige Regularien für die Diözesansynode gibt: So heißt es in der Rubrik des Gesamttextes: compresbyteris nostris Aurelianensis parrochiae sacerdotibus. ${ }^{52}$ Und in c. 4 wird befohlen, dass jeder Priester 2-3 Kleriker (oder Laien) aus seiner Pfarrei zur Diözesansynode mitbringen soll. ${ }^{53}$ In c. 20 wird von Schulen gesprochen, die per villas et vicos eingerichtet werden sollen. ${ }^{54}$

Weiter ist zu nennen Bischof Haito von Basel, dessen Kapitulare ebenfalls vor 813 entstand. Hier ist vor allem interessant, dass Haito sein Kapitular sehr eng, manchmal wörtlich an Kapitel aus der Admonitio generalis 789 angeschlossen hat. ${ }^{55} \mathrm{Ka}-$ pitel 13 spricht von einem Kleriker ex aliena parrochia und meint damit eine andere Diözese als die des sprechenden Bischofs. ${ }^{56}$ c. 21 über den Inzest spricht von in sua parrochia und meint damit die Pfarrei. ${ }^{57}$

Das Konzil von Meaux-Paris (845/46) verfügt in c. 51, dass solche Priester, die mit ihren Herren aus anderen Provinzen in »unsere « Diözesen kommen, keine Gottesdienste halten dürfen. ${ }^{58}$ Solche Kleriker, die zur Weihe vorgeschlagen werden, sollen zu den Bischöfen, aus deren parrochia (= Diözese) sie kommen, zurückgeschickt werden. Dort können sie geweiht oder mit einer Epistola formata ausgestattet werden. Dass ein Bischof keinen Kleriker aus einer anderen Diözese aufnehmen darf, verfügt auch c. 15 von Mainz 888. ${ }^{59}$

Die Herausbildung der Diözese als fest umgrenzter Raum kann auch aus der neuen Betonung des Verbots einer Transmigration oder Translation von Bischöfen herausgelesen werden. ${ }^{\mathbf{6 0}}$ Dies geschah im ausgehenden 9. Jahrhundert nicht erst mit der Angelegenheit des Papstes Formosus, ${ }^{61}$ sondern etwa auch auf dem Konzil von Tribur 895, in dessen Kapitel 42 die Transmigration mit dem Inzest verglichen wird. ${ }^{\mathbf{6}}$

Gegen Wanderbischöfe hatte sich schon Bonifatius in der Mitte des 8. Jahrhunderts ausgesprochen, ${ }^{63}$ und als Erzbischof Frothar von Bordeaux 876 aus Bordeaux durch die Normannen vertrieben wurde, hatte er große Schwierigkeiten, in eine andere Stadt als Metropolit versetzt zu werden: Kenner des Kirchenrechts wie Hinkmar von Reims wandten sich heftig gegen diesen Aktion des westfränkischen Königs, der sich aber letztlich durchsetzen konnte. ${ }^{\mathbf{6 4}}$

$$
* * *
$$

46 Weinfurter (2006) 131

47 Vgl. Hartmann (2006) 95-119.

48 Vgl. zu diesen Quellen Rudolf Pokorny, in: MGH Capitula episcoporum 4 (РокоRny 2005) S. 1-67.

49 Ediert in MGH Capitula episcoporum 1 (BRommer 1984) S. 22-42.

Dass der dort als "Ghaerbald I edierte Text kein Kapitular des Bischofs Ghaerbald von Lüttich ist, hat R. Pokorny, in: MGH Capitula episcoporum 4 (Pokorny 2005) S. 93-96 nachgewiesen.

50 MGH Capitula episcoporum 1 (Brommer 1984) S. 27, 16; S. 30, 6; S. 30, 10 und S. 31, 1.

51 Ebd. S. 39, 2 und S. 42, 1 f.
52 Ebd. S. 103, 1

53 Ebd. S. 106, $11 \mathrm{f}$.

54 Ebd. S. 116, 3.

55 Ebd. S. 204.

56 Ebd. S. 214, 1.

57 Ebd. S. 217, 5. - Siehe dazu unten S. 181.

58 MGH Concilia 3 (Hartmann 1984) S. 109.

59 MGH Concilia 5 (Hartmann u. a. 2012) S. 260, 8-10.

60 Vgl. dazu Scholz (1992), bes. 102-170.

61 Vgl. dazu ZimmermanN (1961), 47-75, und zuletzt Herbers (2012) $100 \mathrm{f}$.
62 MGH Concilia 5 (Hartmann u. a. 2012) S. 364, $10 \mathrm{f}$.

63 Vgl. vor allem Concilium Germanicum (742) c. 4, MGH Concilia 2,1 (Werminghoff 1906) S. 3, 24-26. 64 Vgl. Scholz (1992) $147 \mathrm{ff}$. 


\section{Dekanate}

Die Diözesen wurden seit der Mitte des 9. Jahrhunderts in kleinere Einheiten gegliedert; es entstanden die Dekanate als Amtsbezirke des Dekans oder des Archipresbyters. ${ }^{65}$ Stefan Weinfurter hat im Anschluss an ältere Forschungen behauptet, die Archidiakonate seien erst im ausgehenden 11. Jahrhundert entstanden. ${ }^{66}$ Vielleicht gelten diese Aussagen für das Gebiet östlich des Rheins; im Gebiet des späteren Frankreich und in Oberitalien sind jedenfalls schon in der Mitte und der zweiten Hälfte des 9. Jahrhunderts deutliche Anzeichen für eine Untergliederung der Diözesen in Dekanate zu erkennen. So wird in c. 3 von Toulouse (844) verfügt, dass die Priester, die fünf Meilen im Umkreis um den Bischofssitz amtieren, direkt dem Bischof unterstellt sein sollen, während diejenigen, die weiter entfernt wohnen, einem Archipresbyter zugeordnet werden sollen. ${ }^{\mathbf{6 7}}$

Dekan, Archipresbyter und Archidiakon sind wahrscheinlich nur verschiedene Namen für ein und denselben Amtsträger; ein in der Kanonessammlung des Regino von Prüm überlieferter Kanon bietet die Gleichung decani = archipresbyteri parochiarum. ${ }^{68}$ In den großen Diözesen nördlich der Alpen wurden Visitationen auch durch den Archipresbyter vorgenommen. Das 5. Bischofskapitular Hinkmars von Reims aus dem Jahr 874 bezieht sich fast ganz auf diese Tätigkeit. ${ }^{69}$

Schon Hinkmars 2. Bischofskapitular ist an die Landdekane gerichtet: Die Dekane erhalten eine Liste von Inquisitionsfragen, deren Ergebnisse an den Reimser Metropoliten geschickt werden sollen. Es geht dabei vor allem um den Besitz der Kirche, um die Altartücher, um Kreuze und Reli- quien, um Bücher, Priestergewänder, Chrisma und heiliges Öl und ihre Aufbewahrung und anderes. ${ }^{70}$

Auch die Synode von Pavia 850 kennt Archipresbyter (in c. 6). Sie sollen in den einzelnen Dörfern mit den Familienoberhäuptern zusammenkommen und sie wegen eventueller Vergehen und ihrer Buße befragen. Zur besseren Überwachung der Gläubigen seiner Diözese soll der Bischof einen archipresbyter municipalis und andere ministri einsetzen. ${ }^{71}$ Der Raum der Diözese soll auf diese Weise besser kontrolliert werden.

In verschiedenen Bischofskapitularien des 9. Jahrhunderts werden die so genannten Kalendenkonferenzen erwähnt. Demnach sollen an jedem ersten Tag eines Monats die Presbyter eines Dekanats zusammenkommen. Sie sollen sich dabei nicht dem Essen und Trinken hingeben, sondern sich austauschen über ihr Amt und ihre religiöse Einstellung und über Vorfälle in ihren Pfarreien. ${ }^{72}$ Damit sind wir bei der kleinsten Einheit der kirchlichen Organisation, nämlich bei der Pfarrei.

\section{Pfarrei}

Die umfassendste Bedeutungsbreite unter den hier zu untersuchenden Begriffen nahm der Begriff parrochia an; er kann sowohl Kirchenprovinz, Diözese, Gau als auch Pfarrei bedeuten. ${ }^{73}$ Nach Auffassung von Stefan Weinfurter hat sich im Investiturstreit der Wandel von der »Personalpfarrei« zur »Regionalpfarrei« vollzogen, er schreibt nämlich:

Die frühere Personalpfarrei war im Prinzip über die Personenverbände von Grundherrschaften definiert gewesen. Seit dem späteren 11. Jahr-
65 Vgl. Sägmüller (1898); FAure (1911); PLÖCHL (1953) 345 ff.

66 Weinfurter (2006) 131. Diese Ansicht gründet vielleicht auf den Untersuchungen von Gescher (1919) $90 \mathrm{ff}$., wonach »die ältesten sicheren Zeugnisse für den kölnischen Dekanat « »aus dem 11. Jahrhundert" stammen. Vgl. auch Ahlhaus (1929), bes. 52: „Der Konstanzer Landdekanat ist nicht in fränkisch-karolingischer Zeit entstanden, er stellt sich vielmehr als ein Produkt des 12. Jh. dar. Seine Existenz ist erstmals 1130 urkundlich bezeugt «.
67 MGH Concilia 3 (Hartmann 1984) S. $20 \mathrm{f}$.

68 Es handelt sich um Reginos Kapitel 295 des 1. Buchs seines Sendhandbuchs, das er dem Paenitentiale Theodors von Canterbury entnommen hat, vgl. WASSERSCHLEBEN (1840) 136 mit Anm. p.

69 Vgl. Hinkmar V c. 2 und c. 4, MGH Capitula episcoporum 2 (PoKORNY / STratmanN 1995) S. 87.

70 MGH Capitula episcoporum 2 (Pokorny / Stratmann 1995) S. 45-70.

71 MGH Concilia 3 (Hartmann 1984) S. 222.
72 Vgl. Hinkmar I c. 15, MGH Capitula episcoporum 2 (PokoRnY/ Stratmann 1995) S. 42 f.); Riculf von Soissons c. 22 (ebd. S. 110 f.).

73 Vgl. das Register zu MGH Concilia 3 (Hartmann 1984) S. 613, wo diese Bedeutungen voneinander getrennt sind und das Register zu MGH Concilia 4 (Hartmann 1998), wo (auf S. 715) diese Unterschiede nicht berücksichtigt sind. 
hundert wurde sie abgelöst durch die Regionalpfarrei. Deren Zuständigkeit wurde nun durch eine fest umrissene Region bestimmt. ${ }^{74}$

Wir wollen dazu fragen, ob nicht die Pfarrei bereits in karolingischer Zeit eine »fest umrissene Region« gewesen ist. Nach Karl dem Großen wird die Pfarrei nämlich durch die Zehnten konstituiert. So heißt es in einem Kapitular von 810813 (c. 10): Ut terminum habeat unaquaeque ecclesia, de quibus villis decimas recipiat. ${ }^{75}$ Eher personenbezogen wurde in Chalon 813 (c. 19) bestimmt: "Dorthin sollen die Familien ihre Zehnten geben, wo ihre Kinder getauft werden und wo sie das ganze Jahr hindurch die Messe hören ${ }^{76}{ }^{76}$

Und in einem wahrscheinlich in der Mitte oder der zweiten Hälfte des 9. Jahrhunderts entstandenen Kanon heißt es:

Die Priester sollen an Sonn- oder Feiertagen, bevor sie die Messe zelebrieren, die Gemeinde fragen, ob ein Pfarrkind eines anderen Priesters in der Kirche ist, das dort Messe hören will, weil es seinen eigenen Priester verachtet. Wenn sie ein solches finden, sollen sie es sofort aus der Kirche werfen und dazu bewegen, in seine eigene Pfarrei (parrochia) zurückzugehen. ${ }^{77}$

Hier ist sowohl eine Bindung des Pfarrkindes an die Person des Priesters als auch an den Raum der parrochia (= Pfarrei) angesprochen.

Aus einer Reihe von Texten der 2. Hälfte des 9. Jahrhunderts geht hervor, dass in vielen Fällen mit parrochia ein Raum beschrieben wird, so etwa, wenn von ecclesia parrochiae, ${ }^{\mathbf{7 8}}$ von parrochia rusticana $^{\mathbf{7 9}}$ oder von presbyter parrochiae ${ }^{\mathbf{8 0}}$ die Rede ist.

Kurz vor der Mitte des 9. Jahrhunderts stellten die Pfarreien eine feste Größe innerhalb der kirchlichen Organisation dar. Das bezeugt z. B. Kapitel 7 von Toulouse (844), in dem verboten wurde, Pfar- reien aufzuteilen. Hier wird übrigens zur eindeutigen Bezeichnung von Pfarrei der Begriff parrochia presbyterorum verwendet. ${ }^{\mathbf{8 1}} \mathrm{Im}$ weiteren Verlauf dieses Kapitels folgen dann sehr interessante Ausführungen über die Größe der Pfarreien: Wenn Bedarf besteht (necessitas populi), sollen neue Kirchen gegründet werden,

wenn es die Entfernung erfordert oder die Gefahr des Wassers oder des Waldes ..., dass das Volk oder das schwache Geschlecht, nämlich die Frauen und Kinder, oder die Gebrechlichkeit der Alten nicht zur Hauptkirche (ecclesia principalis) kommen kann ... und wenn der Priester nicht ohne Gefahr rechtzeitig zur Kirche kommen kann, dann soll ein neuer Altar errichtet werden. ${ }^{\mathbf{8 2}}$

Hinkmar von Reims hat dies in seiner Schrift De ecclesiis et capellis ganz ähnlich formuliert:

Solche neuen Bethäuser sollen für das Volk erbaut werden wegen der Gewässer, die zur Winterszeit anzusteigen pflegen, oder wenn vielleicht ein Wald $\mathrm{zu}$ durchqueren ist oder ein Sumpf, oder wenn der Weg so weit ist, dass schwangere Frauen und Kranke zur Metropolitankirche nicht kommen können ... ${ }^{\mathbf{8 3}}$

Die eindeutige Abgrenzung der Pfarreien wird auch im Bischofskapitular Theodulfs von Orléans deutlich, wenn er in c. 14 von der parrochia alterius presbyteri spricht. ${ }^{\mathbf{8 4}}$ Dieser Text ist vom Konzil von Mainz 852 in c. 17 wörtlich rezipiert worden. ${ }^{85}$

Ein festes Band zwischen Pfarrer und Pfarrei wird auch in anderen Bischofskapitularien des beginnenden 9. Jahrhunderts festgelegt, so, wenn Ghaerbald von Lüttich (III c. 7) davon spricht, dass ein Priester nur eine einzige Kirche innehaben dürfe. ${ }^{86}$ Diese Forderung ist in einem in der Mitte
74 WeInfurter (2006) 131.

75 MGH Capitularia 1 (Boretius 1883) S. 178.

76 MGH Concilia 2,1 (WerminghofF 1906) S. 277, 28-30.

77 MGH Concilia 5 (Hartmann u. a. 2012) S. 586, 15-18.

78 MGH Concilia 4 (Hartmann 1998) S. $185,18$.

79 Ebd. S. 470, 39; S. 503, 32; S. 567, 4 f. und S. 598, 40.

80 Ebd. S. 359, 31
81 MGH Concilia 3 (Hartmann 1984) S. 22, 9.

82 Ebd. S. 22, 10-16.

83 MGH Fontes iuris Germanici antiqui 14 (Stratmann 1990) S. 75, 7-10.

84 MGH Capitula episcoporum 1 (Brommer 1984) S. 112, $10 \mathrm{f}$.

85 MGH Concilia 3 (Hartmann 1984) S. 251, 6-13.

86 MGH Capitula episcoporum 1 (Brommer 1984) S. 39, 1-3. 
oder 2. Hälfte des 9. Jahrhunderts entstandenen Kanon sehr schön formuliert:

Denn wie ein Bischof nicht mehr als ein Bistum haben kann, und ein Mann nicht mehr als eine Ehefrau, so kann auch ein Priester nur eine Kirche haben. Deshalb soll kein Priester es wagen, mehrere Kirchen zu haben, es sei denn, er hat in einer jeden andere Priester unter sich, die Tag und Nacht den Gottesdienst feierlich verrichten und in täglichen Zeremonien die Feier der Messe abhalten. ${ }^{87}$

Nach Ghaerbald III c. 18 darf kein Kleriker aus einer fremden Diözese aufgenommen werden. ${ }^{\mathbf{8 8}}$ Gegen wandernde Priester hat sich Haito c. 18 gewandt: nullus ... migret de sua parrochia ad aliam. ${ }^{\mathbf{8 9}}$ Schon die Admonitio generalis 789 hatte in ihrem Kapitel 24 dieses Verbot der alten Kirche (Chalkedon c. 5 und Serdika c. 1) wiederholt. ${ }^{90}$

Auch im 2. Bischofskapitular Hinkmars von Reims (c. 1) ist der enge Zusammenhang von Priester und Dorf deutlich erkennbar, wenn es heißt: in qua villa ... praetitulatus sit presbyter. ${ }^{91}$ Und nach c. 1 des dritten Bischofskapitulars Hinkmars soll über die Büßer in den einzelnen Pfarreien verhandelt werden. ${ }^{\mathbf{9 2}}$

\section{Pfarrkirchen}

Auch über den Raum der Kirche, über das Kirchengebäude, erfahren wir etwas aus den Bischofskapitularien, so wenn Theodulf davon spricht (c. 8), dass häufig in den Kirchen die Ernte und Heu gelagert werden (crebro in ecclesiis messes et fenum congeri). ${ }^{93}$ Nach c. 10 soll die Kirche nur dem Gottesdienst dienen; es soll dort keine anderen Zusammenkünfte, kein Lachen und keine überflüssigen Gespräche geben. ${ }^{94}$ Im Altarraum einer Kirche dürfen sich nach c. 6 Frauen nicht aufhalten: nequaquam ad altare accedant, sed locis suis stent. ${ }^{95}$ Sie haben in der Kirche vorgeschriebene Plätze.

Über das Aussehen des Kirchenraums hat sich Hinkmar in seinem 2. Bischofskapitular geäußert (c. 13): qualiter sit cooperta ecclesia aut si sit camerata. ${ }^{96}$ Das heißt doch, die Art der Dachdeckung und die innere Einteilung des Kirchengebäudes soll untersucht werden. Was die äußere Gestalt des Kirchengebäudes angeht, so spricht Hinkmar vom Atrium und sagt (Hinkmar II, c. 15): si atrium habeat munitum aut cellam propriam iuxta ecclesiam, d. h. ob das Atrium ein fester Bauteil ist und ob der Priester ein eigenes Wohngebäude neben der Kirche hat. ${ }^{97}$ Etwas abgewandelt erscheint diese Vorschrift im Sendhandbuch Reginos von Prüm I, Fragenkatalog Nr. 16: "Auch ist zu beachten, dass der Vorraum der Kirche durch einen Zaun gesichert ist, damit er nicht verunreinigt werden kann. ${ }^{98}$

Wie es um eine Landkirche herum aussieht, wird von Hinkmar angesprochen, wenn er (II c. 2) von mansus praeter cimiterium et curtem, ubi ecclesia et domus ipsius (scil. presbyteri) continetur spricht: also vom Ackerland, dem Friedhof und dem Hof, auf dem das Kirchengebäude und das Haus des Priesters stehen. ${ }^{99}$ Ganz ähnlich heißt es bei Regino in Buch I, Fragenkatalog Nr. 14:

Es muss untersucht werden, ob diese Kirche eine Hofstelle von 12 bunuaria (= ca. 3 Morgen, d. h. 30 ar) besitzt - außer dem Friedhof und dem Hof, wo die Kirche und das Haus des Priesters stehen - und ob sie vier Hörige hat. ${ }^{\mathbf{1 0 0}}$

Auch für die Überwachung der Gläubigen war die Pfarrei ein ganz wichtiger Raum:
87 MGH Concilia 5 (Hartmann u. a. 2012) S. 584, 17-21.

88 MGH Capitula episcoporum 1 (Brommer 1984) S. 42, $1 \mathrm{f}$.

89 Ebd. S. 216, $1 \mathrm{f}$

90 MGH Fontes iuris Germanici antiqui 16 (Mordek u. a. 2012) S. 196.

91 MGH Capitula episcoporum 2 (Pokorny/Stratmann 1995) S. 45, $10 \mathrm{f}$.

92 Ebd. S. 73 mit Anm. 10.

93 MGH Capitula episcoporum 1 (Brommer 1984) S. 108, 7.
94 Ebd. S. 110.

95 Ebd. S. 107, 6 f.

96 MGH Capitula episcoporum 2

(Pokorny / Stratmann 1995) S. 49, $1 \mathrm{f}$.

97 Ebd. S. 49, $4 \mathrm{f}$.

98 Wasserschleben (1840) 20.

99 MGH Capitula episcoporum 2

(Pokorny / Stratmann 1995) S. 46, $1 \mathrm{f}$.

100 WASSERSCHLEBEN (1840) 20. 
So heißt es in einem Kanon, den Regino dem Konzil von Rouen zuschreibt:

Das Volk soll ermahnt werden, sich an Sonnund Feiertagen auf jeden Fall vollzählig bei der Vesper und den nächtlichen Vigilien und bei der Messe einzufinden; und es sollen aufrichtige und gottesfürchtige Männer als Dekane in den Städten und Pfarrgemeinden eingesetzt werden, die die Müßigen und Nachlässigen ermahnen, ohne Ausrede zum Gottesdienst zu kommen. Diese Dekane selbst sollen durch einen Eid verpflichtet werden, die Nachlässigen und Übertreter keinesfalls zu verschweigen - weder aus Liebe noch aus Furcht, Verwandtschaft oder Bestechlichkeit -, sondern ihre Verfehlung ihren Priestern zu offenbaren ... ${ }^{\mathbf{1 0 1}}$

$$
* * *
$$

Zentralität wurde hier nicht nur untersucht im Großen und Ganzen des Reiches oder der Kirchen- provinz, sondern vor allem in den kleineren Einheiten von Diözese und Pfarrei.

Neben dem immer wieder zitierten Hinkmar von Reims war es vor allem Theodulf von Orléans, der für die Gestaltung und die Gestalt von bischöflicher Diözese und priesterlicher Pfarrei grundlegende Äußerungen formuliert hat. Da Theodulfs erstes Bischofskapitular eine weite Verbreitung erfahren hat, ${ }^{\mathbf{1 0 2}}$ kann sein Einfluss auf die Gestaltung des kirchlichen Raumes kaum überschätzt werden.

Auch die Bewegung im Raum ist von Bedeutung für die Ausgestaltung des kirchlichen Raums im frühen Mittelalter. Dabei ist vor allem an die jährliche Visitationsreise des Bischofs zu denken, später auch an das Sendgericht, das an wechselnden Orten in der gesamten Diözese, eigentlich in allen Pfarrgemeinden, tagen sollte.

\section{Bibliographie}

- Ahrhaus, Joseph (1929), Die Landdekanate des Bistums Konstanz im Mittelalter. Ein Beitrag zur mittelalterlichen Kirchenund Kulturgeschichte, Stuttgart

- Barion, Hans (1931), Das fränkisch-deutsche Synodalrecht des Frühmittelalters, Bonn

- Boretius, Alfred (Hg.) (1883), Capitularia regum Francorum, (MGH Capitularia 1), Hannover

- Brommer, Peter (Hg.) (1984), Capitula episcoporum, Teil 1, (MGH Capitula episcoporum 1), Hannover

- Diez, Gonzalo Martínez, Felix Rodriguez (Hg.) (1992), La Colección canónica Hispana, Bd. 5, Madrid

- Epp, Verena (1998), Zur Kategorie des Raumes in frühmittelalterlichen Rechtstexten, in: Raum und Raumvorstellungen im Mittelalter, hg. v. Aertsen, Jan A., Andreas Speer, Berlin, 575-590

- Faure, Joseph (1911), L'archiprêtre, des origines au droit décrétalien, étude d'histoire et de droit eccléstiastiques. Thèse de doctorat en droit canonique, Lyon

- Gescher, Franz (1919), Der kölnische Dekanat und Archidiakonat in ihrer Entstehung und ersten Entwicklung. Ein Beitrag zur Verfassungsgeschichte der deutschen Kirche im Mittelalter, Stuttgart

- Hartmann, Wilfried (Hg.) (1984), Die Konzilien der karolingischen Teilreiche 843-859, (MGH Concilia 3), Hannover

- Hartmann, Wilfried (Hg.) (1998), Die Konzilien der karolingischen Teilreiche 860-874, (MGH Concilia 4), Hannover

- Hartmann, Wilfried (2006), »Sozialdisziplinierung« und »Sündenzucht« im frühen Mittelalter? Das bischöfliche Sendgericht in der Zeit um 900, in: Jahrbuch des historischen Kollegs 2005, 95-119

- Hartmann, Wilfried u.a. (Hg.) (2012), Die Konzilien der karolingischen Teilreiche 875-911, (MGH Concilia 5), Hannover

- Hauck, Albert (1935), Kirchengeschichte Deutschlands, Bd. 2, 5. Aufl., Leipzig

- Herbers, Klaus (2012), Geschichte des Papsttums im Mittelalter, Darmstadt

101 MGH Concilia 5 (Hartmann u. a. 2012) S. 591, 24-30.

102 Vgl. MGH Capitula episcoporum 1 (Brommer 1984) S. 76 ff., wo 49 Handschriften des Kapitulars verzeichnet sind. Im 4. Teil (MGH Capitula episcoporum 4, PoKorny 2005) sind auf S. 77 ff. weitere 4 Handschriften nachgetragen. 
- Hinschius, Paul (1883), System des katholischen Kirchenrechts mit besonderer Rücksicht auf Deutschland, Bd. 3, Berlin

- Manssen, Friedrich (Hg.) (1893), Concilia aevi Merovingici, (MGH Concilia 1), Hannover

- Mordek, Hubert u. a. (Hg.) (2012), Die Admonitio generalis Karls des Großen, (MGH Fontes juris XVI), Hannover

- Pangerl, Daniel Carlo (2011), Die Metropolitanverfassung des karolingischen Frankenreiches, Hannover

- Plöchl, Willibald Maria (1953), Geschichte des Kirchenrechts 1: Das Recht des ersten christlichen Jahrtausends. Von der Urkirche bis zum Großen Schisma, München

- Pokorny, Rudolf (Hg.) (2005), Capitula episcoporum, Teil 4, (MGH Capitula episcoporum 4), Hannover

- Pokorny, Rudolf, Martina Stratmann (Hg.) (1995), Capitula episcoporum, Teil 2, (MGH Capitula episcoporum 2), Hannover

- Sägmüller, Johannes Baptist (1898), Die Entwicklung des Archipresbyterats und Dekanats bis zum Ende des Karolingerreiches Tübingen

- Scholz, Sebastian (1992), Transmigration und Translation. Studien zum Bistumswechsel der Bischöfe von der Spätantike bis zum Hohen Mittelalter, Köln

- Stratmann, Martina (1990), Hinkmar von Reims, Collectio de ecclesiis et capellis, (MGH Fontes iuris Germanici antiqui 14), Hannover

- Vives, José (Hg.) (1963), Concilios Visigóticos e Hispano-Romanos, Madrid

- Wasserschleben, Friedrich Wilhelm Hermann (Hg.) (1840), Regino von Prüm, Libri duo de synodalibus causis et disciplinis ecclesiasticis, Leipzig

- Weinfurter, Stefan (2006), Canossa. Die Entzauberung der Welt, München

- Werminghoff, Albert (Hg.) (1906), Concilia aevi Carolini 742-842, (MGH Concilia 2,1), Hannover

- Zimmermann, Harald (1961), Papstabsetzungen des Mittelalters. 1. Teil: Die Zeit der Karolinger, in: Mitteilungen des Instituts für Österreichische Geschichtsforschung 69, 1-84 